\title{
Ansätze zu einer emanzipatorischen Familienpolitik: Der Siebte Familienbericht
}

Familienpolitik wird derzeit vom weichen zum harten politischen Thema. Dies ist vor allem auf die vielfältigen Konsequenzen des demografischen Wandels zurückzuführen. Die Gleichstellungspolitik führt hingegen ein Schattendasein. Der Siebte Familienbericht der Bundesregierung, der als zentrales Dokument der aktuellen Familienpolitik gelesen werden kann, versucht, eine Brücke zwischen den Politikbereichen zu schlagen. Er argumentiert, dass Familienpolitik die Geschlechterfrage nicht überspringen kann. Die Schnittmengen zwischen einer modernisierten Familienpolitik und Gleichstellungspolitik sind groß, machen aber eine eigenständige Gleichstellungspolitik mitnichten überflüssig.

\section{Familie im Zentrum, Gleichstellung am Rand?}

Seit einigen Jahren erlebt Deutschland eine neue und breite Aufmerksamkeit für Familienpolitik. Thematisch beschränkt sie sich nicht länger auf den klassischen Kanon einer weitreichenden Familienförderung durch eine breite Palette finanzieller Transferleistungen, vereinzelte, eher unkoordinierte Maßnahmen sowie Appelle zu feierlichen Anlässen. Auch die Akteursebene von Bund, Wohlfahrtsverbänden und Kirchen wird erweitert: Repräsentanten aus Wirtschaft, Politik und Zivilgesellschaft auf den Ebenen von Bund, Ländern und Kommunen beteiligen sich in breiter Allianz. Fast noch erstaunlicher ist jedoch, dass Themen, die seit 30 Jahren auf der Agenda einer emanzipatorischen Familienpolitikforschung stehen (Jurczyk 1976), derzeit Eingang in den Mainstream der bundespolitischen Familienpolitik finden. Was ist geschehen, dass die Familienpolitik vom Rand ins Zentrum der politischen Debatten rückt, vom „weichen“ zum „harten“ politischen Thema avanciert - ganz im Gegensatz zur Gleichstellung der Geschlechter? Denn Themen wie Lohnungleichheit oder Gewalt in der Ehe taugen längst nicht mehr für Schlagzeilen.

\section{Umgewichtungen und Hintergründe}

Der Auslöser ist schlicht. Nicht das Ziel einer feministischen Familienpolitik treibt zum Umdenken. Alarmierend wirkt vielmehr der Geburtenrückgang, der Themen wie Ganztagsbetreuung, eine Umdefinition der „guten“ Mutter sowie auch die Beteiligung von Vätern an der Kinderbetreuung durch das Novum der „Vätermonate“ endlich hoffähig werden lässt. Die Debatte wird begleitet von vielfältigen, mehr oder weniger lauten, medial aufbereiteten Krisenszenarien rund um Familie. Bedenkt man jedoch, dass die Geburtenrate in Deutschland bereits seit den 1970er Jahren bei 1,3 bis 1,4 Kindern pro Frau liegt (Klein 2005, S. 62f.), so müssen umfassendere Konsequenzen der schrumpfenden Bevölkerung zur politischen Bewusstwerdung beigetragen haben. $\mathrm{Zu}$ ihnen gehören: der sich abzeichnende Mangel an qualifizierten Arbeitskräften, die begrenzten Möglichkeiten, diesen Mangel - ohne drastische Änderungen in der Einwanderungs- und Integrationspolitik - durch ausländische Arbeitskräfte zu kompensieren, die Gleichzeitigkeit von Geburtenrückgang und deutlich zunehmender Lebenserwartung, die eine umfassende Neu-Konstruktion der sozialen Sicherungssysteme erfordert; aber auch die Folgen streitbarerer Geschlechterverhältnisse, die das Beziehungs- und Heiratsverhalten von Frauen ändern, die Entleerung von Regionen und die sozialräumlichen wie auch infrastrukturellen Folgen, wenn sich Generationenverhältnisse verschieben (Kaufmann 2005). Hinter dem scheinbar konservativen Ruf nach mehr Kindern steht folglich die Erkenntnis, dass sich ändernde familiale, geschlechterbezogene und generative Verhältnisse - die allerdings durchaus selber bereits zum Gegenstand diskursiver Auseinandersetzungen werden (Hondrich 2006; Auth/Holland-Cunz 2007) - Auswirkungen für die Gesellschaft als Ganze haben. Mithin ist im Zuge einer übergreifenden Weiterentwicklung der Moderne hin zur späten Moderne (Beck 1986) die scheinbar natürliche Selbstverständlichkeit dieser Verhältnisse nicht nur ins Rutschen geraten, sondern im doppelten Wortsinn reflexiv geworden. Familie wird einschließlich ihrer lange inhärenten traditionellen Geschlechterverhältnisse von der „Naturressource“ zur alltäglichen und biografischen Herstellungsleistung (Jurczyk/Schier 2007), die permanenter bewusster Gestaltung bedarf. Ein solches „Doing Family“ ist eine keinesfalls selbstverständliche, sondern zunehmend voraussetzungsvolle Aktivität derjenigen Frauen, Männer, Kinder, Jugendlichen, die in Familien leben und sich dabei stets mit den gesellschaftlichen Rahmenbedingungen auseinandersetzen müssen. Sie ist - neben ihrer emotionalen Bedeutung auch ein Stück Arbeit, das Ressourcen bindet. Dies bedeutet auch eine späte gesellschaftliche Anerkennung der Relevanz des Theorems der Frauen- und Geschlechterforschung zum Zusammenhang von gesellschaftlicher und individueller Reproduktion (Becker-Schmid 2004). Neu ist, dass Hausarbeit, reproduktive Arbeit, Fürsorgearbeit und damit Familie aus ihrer Unsichtbarkeit heraustreten und in ihren unverzichtbaren, aber mittlerweile erodierenden Leistungen für die Gesellschaft expliziert werden.

Karin Jurczyk, Dr., Leiterin der Abteilung Familie und Familienpolitik im Deutschen Jugendinstitut e. V., Arbeitsschwerpunkte: Familie, Familienpolitik, Gender, Arbeit, Zeit, Lebensführung e-mail: jurczyk@dji.de 
Mit dem Ansatz der Familienrhetorik (Lange et al. 2000) werden die vielfältigen Diskurse um Familie rekonstruierbar und interpretierbar. Angesichts der Veränderungsprozesse ringen unterschiedliche Akteure um diagnostische und normative Definitionen dessen, was Familie ist, was und wie sie sein soll. Entscheidend in diesem Kampf um Definitionsmacht ist für eine emanzipatorische Familienpolitik, Familie als historisch und kulturell wandelbares System persönlicher fürsorgeorientierter Generationen- und Geschlechterbeziehungen zu betrachten, das sich zudem immer wieder hinsichtlich seiner Zusammensetzung, Leistungen und Bedeutung für seine Mitglieder und für die Gesellschaft verändert. Stellt man private fürsorgliche Beziehungen zwischen Generationen und Geschlechtern in den Mittelpunkt des Familienbegriffs, so wird zum einen die Vielfalt weit über die Normalfamilie hinaus sichtbar (convergence to diversity). Zum anderen erscheint die historisch gewachsene Konnotation mit traditionellen Geschlechterrollen als auflösbar und damit als Gestaltungsaufgabe von Politik. Aus derartigen historisch-prozessualen Interpretationen von Familie ergeben sich Begründungen für eine Familienpolitik, die egalitäre Geschlechterverhältnisse fördert und auf entsprechend gendersensible Unterstützungsleistungen für Familien bzw. deren Unterlassung setzt.

In diese Richtung lässt sich der Siebte Familienbericht der Bundesregierung (BMFSFJ 2006) interpretieren: Er ist ein Ansatzpunkt für ein familienpolitisches Konzept, das versucht, die Gleichstellung der Geschlechter systematisch zu integrieren und hierfür einige zentrale Bausteine benennt. Diese Konzeptualisierung entlang eines Leitgedankens ist bereits ein Novum; erst Recht die Orientierung an Geschlechterfragen. In diesem Sinn bricht der Bericht mit der Praxis, Familien- und Gleichstellungspolitik einander gegenüber zu stellen, sondern trägt zu ihrer partiellen Integration bei. Dieser Ansatz soll im Folgenden nachvollzogen (Abschnitt 3), um vorgeschlagene politische Szenarien ergänzt (Abschnitt 4) sowie abschließend auf seine Grenzen hin (Abschnitt 5) diskutiert werden.

\section{Konzeptuelle Bausteine des Siebten Familienberichts}

\subsection{ECKPUNKTE}

Familienberichte werden seit 1965 im Auftrag des Bundestages regelmäßig, d.h. mindestens in jeder zweiten Legislaturperiode, erstellt. Verantwortlich ist eine bis zu siebenköpfige Sachverständigenkommission, die vom Bundesfamilienministerium eingesetzt und durch eine Geschäftsführung im Deutschen Jugendinstitut e. V. unterstützt wird. Den Familienberichten wird eine Stellungnahme der Bundesregierung angefügt. Sie dienen nicht nur der Politikberatung, wie ursprünglich mit dem Auftrag zur Berichterstattung intendiert, sondern strahlen mittlerweile stark auf die öffentliche und fachpolitische Diskussion zu Familien aus.

Die „Zukunft von Familie“ war das Leitthema des Siebten Familienberichtes (BMFSJ 2006). Damit rückten die Identifikation von Problemfeldern, ihre inner- und außerfamilialen Dynamiken, einschließlich des säkularen Trends des Geburtenrückgangs, in den Mittelpunkt. Sie wurden - nicht zuletzt aufgrund der Zusammensetzung der Kommission - anhand der Eckpunkte Ökonomie, Fürsorge und Geschlechterpartizipation akzentuiert. Unweigerlich führte die Entscheidung, erstmals systematisch europäische Erfahrungen einzubeziehen (ebd., Kap. II), dazu, den Zusammenhang zwischen der Modernität der Geschlechterverhältnisse und der der Familienpolitiken in Europa sichtbar zu machen. Die sehr unterschiedlichen Pfade von Familienpolitik in Dänemark, Frankreich, Vereinigtem Königreich, Niederlanden und Deutschland haben klar gezeigt, dass sich eine hohe Frauenerwerbstätigkeit, gute und ausreichende Kinderbetreuung mit einer höheren Geburtenrate verbinden lassen. Eine integrierte Analyse der Geschlechterverhältnisse durchzieht den Bericht in vielen Kapiteln wie ein roter Faden. Denn ohne diese ließen sich weder der Wandel von Familie und die „Knappheit“ von Fürsorge als Ressource (ebd. Kap. III), die veränderten Dynamiken im innerfamilialen Beziehungsgeschehen (ebd. Kap. IV) noch die Probleme der Zeitorganisation (ebd., Kap. VI) hinreichend erklären.
Zentrale Einsicht ist ein Modernisierungsdefizit der Familienpolitik in Deutschland mit fatalen Folgen: Die Beibehaltung traditionell gerahmter Geschlechterverhältnisse führt bei sich gleichzeitig verändernden gesellschaftlichen Bedingungen zu erheblichen Problemen im Familienleben und verhindert nicht zuletzt auch die Realisierung von Kinderwünschen. Allerdings standen die Themen „Kinderwunsch“ und „Erhöhung der Geburtenrate" weder analytisch noch politisch im Zentrum des Berichtes. Die Forschung desillusioniert ohnehin die Vorstellung simpler Stellschrauben oder Blaupausen für eine geburtenfördernde Politik, die sich ohne Weiteres von einem Land auf das andere übertragen ließen (Björklund 2007; McDonald 2000). Der Bericht argumentiert deshalb eher in die Richtung, dass strukturelle Hindernisse für ein menschlich befriedigendes, geschlechtergerechtes und gesellschaftlich funktionales Familienleben ausgeräumt und Gelegenheitsstrukturen für Sozialisation, Fürsorge und Reproduktion geschaffen werden sollten, was ggf. die Geburtenraten mittelbar beeinflussen kann.

\subsection{ARGUMENTATIONSFIGUREN}

Die Analysen des Familienberichts sind multifaktoriell. Folgende, eng zusammenhängende gleichstellungsrelevante Aspekte lassen sich hieraus zu Argumentationsfiguren bündeln:

\section{TEILHABE BEIDER GESCHLECHTER}

Erstens wird die alltägliche und biografische Teilhabe beider Geschlechter an Fürsorgebeziehungen und weiteren gesellschaftlichen Bereichen - allen voran am Erwerbsbereich - als Integrationsmodell in Gesellschaft entwickelt. Dies basiert weder auf einem voluntaristischen noch einem moralischen Ansatz, sondern auf einem Verständnis demokratischer Praxis von Bürgerrechten als Teilhaberechte und -pflichten für alle (Tronto 2000). Das auf Erwerbsarbeit reduzierte ,adult-workermodel" wird erweitert um verlässliche Fürsorgeleistungen im Lebenszusammenhang und Lebensverlauf. Dies geschieht nicht nur mit Blick auf die Wünsche vieler Frauen und Männer nach einem solchen Lebensmodell (Cornelißen 2006), sondern auch, um die Armutsgefahr für Frauen und Kinder in einer sich verändernden Erwerbsgesellschaft zu verringern (Meier et al. 2003). 


\section{FAMILIE ALS NETZWERK}

Zweitens wird Familie als multilokales Netzwerk gesehen. Will man heutige und zukünftige Familien angemessen verstehen, so ist nicht nur die gängige Konnotation von Familie mit Ehe und traditionellen Geschlechterrollen aufzulösen, sondern auch diejenige, die Familie mit Haushalt gleichsetzt (Bien/Marbach 2003). Multilokale familiale Fürsorgebeziehungen umfassen mehr als Eltern und ihre kleinen Kinder. Sie berücksichtigen auch die Fürsorge erwachsener Kinder für ihre alten Eltern und weitere Verwandte. Familie ist nicht an das gemeinsame tägliche Leben in einem Haushalt gebunden. Sie ist als Lebens- und Lernzusammenhang ein Netzwerk emotionsbasierter, persönlicher Austauschbeziehungen, die nicht einfach als vorhanden vorausgesetzt werden können.

\section{SOZIALHISTORISCHER KONTEXT}

Der Bericht ordnet drittens den Wandel von Familien sozialhistorisch ein: Mit dem Übergang vom fordistischen zum sogenannten postfordistischen Gesellschaftsmodell, mit der Erweiterung nationaler zu globalen Ökonomien, einschließlich der zeitlichen und räumlichen Flexibilisierung der Erwerbswelt sowie wachsender berufsbiografischer Diskontinuitäten, brechen arbeitsteilige Strukturen zwischen Produktions- und Reproduktionssphäre wie auch zwischen den Geschlechtern auf, wenngleich Deutschland im Vergleich nach wie vor den höchsten Anteil nicht erwerbstätiger Mütter aufweist (Eichhorst et al. 2007) und Frauen nach wie vor den größten Teil familialer Arbeit leisten. Reibungsverluste für Familien entstehen zusätzlich durch Ungleichzeitigkeiten: Die Kontextinstitutionen von Familie - Kindergarten und Schule, Behörden und Geschäfte, Firmen, Städtebau und Verkehrswesen - sind noch immer mehrheitlich an fordistisch organisierten Geschlechterverhältnissen ausgerichtet. Dies führt zu ambivalenten Entgrenzungsprozessen zwischen den Sphären Familie und Erwerbswelt, die überwiegend von Frauen ausbalanciert werden müssen (Jurczyk et al. 2005).

\section{LEBENSVERLAUFSPERSPEKTIVE}

Viertens steht die Lebensverlaufsperspektive im doppelten Sinn im Zentrum: als dynamische Veränderung von Familie in ihrem Verlauf sowie als Lebenslaufregime in seiner Bedeutung für Geschlechter- und Familienverhältnisse. Diese manifesten und latenten Strukturierungen der biografischen Zeit bestimmen heute die linear gedachte Lebensablauflogik mit der Abfolge von Herkunftsfamilie, Bildung, Familie für Frauen, Erwerbsarbeit und Verrentung sowie parallel angelegte Lebensbereichsprogramme entlang traditioneller Geschlechterrollen (Krüger 2005, 2006). Deutschland verfügt aufgrund seiner sehr spezifischen Institutionenpraktiken über ein im Vergleich zu anderen modernen Gesellschaften hart gerahmtes Lebenslaufregime, in dem Entscheidungen schwer rückgängig zu machen sind. Geschlechtlich konnotierte Lebenslaufregime sind in Strukturen (Ausbildungsverordnungen, Karrierepfade, Arbeitszeitmodelle) wie in individuellen Selbstkonzepten verankert. Nach wie vor internalisieren Männer eine Ernährerrolle, die kontinuierliche Vollzeitarbeit impliziert. Das in Lebensläufen beider Geschlechter verankerte Vollernährermodell hat jedoch keine Zukunft. Denn es bröckeln seine wichtigsten Voraussetzungen: die hohe Erwerbssicherheit von Männern als Basis für die Rolle des Familienernährers und die Beschränkung von Frauen auf die Hausfrauen- bzw. Zuverdienerrolle. Befristete und andere prekäre Beschäftigungsverhältnisse nehmen zu, Arbeitsmarktsegmente verschieben sich in Richtung weiblich konnotiertes Sozial- und Gesundheitswesen, gleichzeitig konkurrieren zunehmend hoch qualifizierte Frauen mit Männern um die gleichen Arbeitsplätze. Lebenslaufregime stimmen nur noch bedingt mit den realen Verhältnissen und den Wünschen überein: Berufliche und private Lebensverläufe werden von Linien $\mathrm{zu}$ „Mäandern“ und „Jo-Jos“ mit multiplen Erwerbs-, Elternschafts- und Partnerschaftsphasen (Stauber/du Bois-Reymond 2006). Auf diesem Hintergrund verschieben sich Heiratsdatum und Erstgeburt immer weiter nach hinten im Lebensverlauf. Die verdichtete Lebensphase zwischen Ende 20 und Mitte 40 wird zur sogenannten rushhour of life, in der gesellschaftliche Integrationsaufgaben von Fürsorge sowie Erwerb gleichzeitig erbracht werden müssen. Dies wird als ein entscheidender Grund für die Aufschiebung und oft NichtRealisierung von Kinderwünschen angesehen. Auch wenn die jüngere Generation ihre „neue“ flexible Erwerbssituation nicht so negativ beurteilt wie die eher sicher- heitsgewohnte Generation davor, zeigen sich doch europaweit Grenzen der Verarbeitung beruflicher und ökonomischer Unsicherheit sowie geforderter Flexibilität und Mobilität. Insbesondere bei Männern führen die strukturellen Erschwernisse, die männliche Ernährerrolle zu erfüllen, seltener zu innovativen Rollenmodifikationen und häufiger zu einer Verweigerung von Elternschaft (Mills/Blossfeld 2003; Tölke 2004).

\section{ZEITORGANISATION}

Fünftens wird die Zeitorganisation von Familien als wichtige Gestaltungsebene erstmals in einem Familienbericht näher beleuchtet. Ausgehend davon, dass Familie zeitlichen Eigenlogiken folgt und für ihre Konstituierung sowie die Aufrechterhaltung von Beziehung und Fürsorge Zeit braucht, Verfügung über Eigenzeit also eine entscheidende Ressource ist, wird offensichtlich, dass nicht selbstbestimmbare flexible Arbeitszeiten verbindliche Beziehungen und verlässliche Fürsorgeleistungen erschweren. Denn in Familien treffen die unterschiedlichen inneren und äußeren Taktgeber der einzelnen Mitglieder oft konflikthaft aufeinander. Insbesondere die Erwerbsarbeitszeit dominiert die Familienzeit, umso mehr, wenn zwei Elternteile arbeiten. Der Wandel zur flexiblen Dienstleistungsgesellschaft wird als ambivalente Chance auch für neue Geschlechterverhältnisse gesehen, die im Interesse einer geschlechtergerechten Familie dringenden Gestaltungsbedarf aufwirft.

\section{GESELLSCHAFTLICHE INSTITUTIONEN}

Sechstens ist Familie stets im Kontext gesellschaftlicher Institutionen zu sehen. Sie ist zwar Akteur mit eigenen Leistungen, Ressourcen und Handlungspotenzialen an den Schnittstellen zwischen Privatheit und dem Institutionengeflecht verschiedener Öffentlichkeiten, insbesondere auch eingebunden in den sozialen Nahbereich und Nachbarschaft. Durch diese Eingebundenheit ist sie aber angewiesen auf die Leistungen, Berücksichtigung und Anerkennung der mit ihr verknüpften gesellschaftlichen Teilsysteme, die unter postfordistischen Bedingungen immer weniger zusammenpassen. Auch hier geht es also um die Abstimmung zwischen diversen Umweltkontexten auf die Bedarfe von Familien. 


\section{ZWISCHENFAZIT}

Die analytischen Eckpunkte des Berichtes zeigen, dass es dringliche Gründe für eine Modernisierung der Familienpolitik gibt. Die multifaktoriellen Analysen - zu Veränderungen der Bildungs- und Erwerbsverläufe, Umstrukturierungen des Arbeitsmarktes und sozialen Kontextes, Fürsorgedilemmata im Alltag und Lebensverlauf, Normalisierung von Brüchen in privaten Beziehungen, neues Wissen über eine gute Förderung des Nachwuchses einschließlich der Bedeutung sorgender Väterlichkeit - verweisen alle auf eine enge Verknüpfung von Familien- mit Gleichstellungspolitik (Abschnitt 5). Sie führen zum Konsens, dass eine Familienpolitik, die in ihrer Geld-, Zeit und Infrastrukturpolitik weiter auf asymmetrische Geschlechterverhältnisse setzt, Probleme der Gesellschaft, nicht nur der Familien, forciert statt sie zu lösen.

\section{Lösungsansätze: Szenarien}

Vor diesem Hintergrund werden im Siebten Familienbericht Szenarien entwickelt, gerahmt vom Konzept nachhaltiger Familienpolitik sowie den Orientierungspunkten „Flexibilität und Verlässlichkeit“. Nachhaltigkeit meint hier zum einen eine langfristige, kontinuierliche und verlässliche Familienpolitik, zum anderen Rahmenbedingungen, die es der nachwachsenden Generation ermöglichen, in Kinder zu investieren, Generationensolidarität zu leben und Fürsorge für andere als Teil der eigenen Lebensplanung umzusetzen und dabei der Dynamik und Vielfalt familialer Lebenszusammenhänge gerecht $\mathrm{zu}$ werden (Bertram et al. 2005).

Flexibilität und Verlässlichkeit sind qualitative Leitlinien einer solchen Familienpolitik, um auf die Besonderheit familialer Bedarfe reagieren zu können. Denn Familienleben ist einerseits in besonderem Maße auf den flexiblen Umgang mit Zeit und Ressourcen angewiesen. Andererseits aber ist ein Minimum stabiler Rahmenbedingungen zwingend notwendig, damit soziales Leben in Familien überhaupt zustande kommt.

\subsection{SZENARIO ZEIT}

Ohne gemeinsame Alltagszeit und miteinander verschränkbare Lebensläufe können Familien sich weder konstituieren noch als solche leben noch ihre Leistungen erbringen. Zeitstrukturen der Gesellschaft sind auf die Institution Familie auszurichten und nicht umgekehrt.

\section{ALLTAGSZEIT}

Im Focus steht eine Arbeitszeitpolitik, die die Zeitbedarfe der Familien und ihrer Mitglieder ernst nimmt und Flexibilität auch im Interesse von Familien interpretiert. Die große Diskrepanz zwischen Arbeitszeitrealität und Arbeitszeitwünschen gilt es zu schließen (Klenner 2007; Holst 2007). Im Einzelnen geht es um passgenaue und differenzierte Angebote im Sinne eines familydiversity-managements, um die Ermöglichung von Vielfalt in einem definierten Rahmen von Arbeitszeitoptionen, um dynamische Anpassungen im Familienverlauf, um die Einbindung der gesamten Belegschaft und die Ermöglichung von Partizipation sowie nicht zuletzt um eine kontrollierte Flexibilität (BMFSFJ 2006, S. 272ff.). Viele dieser Elemente sind seit Langem in der Diskussion. Es gilt nun, von vereinzelter guter Praxis zu Regelstrukturen und -verfahren in Unternehmen zu kommen und sie mit entsprechenden Unternehmenskulturen zu stützen. Hinzuzufügen wäre, angesichts zunehmender Streßphänomene, eine stärkere Beachtung einer gesundheitserhaltenden Familienpolitik in Betrieben, um die Herstellungsleistungen von Familie nicht weiter zu gefährden (Schier/Lange 2007).

Eine solche Arbeitszeitpolitik wird integriert in eine lokale Zeitpolitik, denn die Zeitraumpfade von Frauen und Männern sind nach wie vor extrem unterschiedlich, auf das fordistische Modell der allzeitverfügbaren Hausfrau und Mutter kann aber nicht mehr selbstverständlich zurückgegriffen werden. Hier bedarf es einer koordinierten Vernetzung der örtlichen Infrastrukturen und insbesondere einer gezielten Abstimmung der öffentlichen Taktgeber Schule und Kindergarten mit Betrieben, einer Optimierung von Wegezeiten sowie wiederum der Partizipation der Betroffenen. Die Zielsetzung ist Zeitwohlstand für Familien und ihre Mitglieder. Instrumente hierfür sind Zeitbüros und Mobilitätspakte, die lokale Zeitanbieter und -nachfrager aufeinander abstimmen.

\section{LEBENSLAUF}

Im Zentrum steht das Aufbrechen des traditionellen, dreiphasigen Lebenslaufs (Kind/Arbeitsmarkt- bzw. Familienteilhabe/Rente). Die anvisierte „Entzerrung des Lebenslaufs " bedeutet zum einen, die vor allem durch tradierte Ausbildungs-, Berufs- und Arbeitsmarktstrukturen - jetzt enorm verdichtete sogenannte „Rushhour of Life", d.h. die Gleichzeitigkeit von Familiengründung und beruflicher Etablierung, zu entdichten. Zum Zweiten müssen Möglichkeiten gefunden werden, um (üblicherweise im späteren Familienverlauf) die Versorgung der Elterngeneration mit eigener Berufstätigkeit vereinbaren zu können. Hierfür steht das Stichwort „Optionszeitenmodell“. Es bedeutet, die Erwerbsarbeit für andere, gesellschaftlich wichtige Careund andere Teilhabeaufgaben unterbrechen zu können. Angeregt durch die niederländische Lebenslaufregelung (DGFZP 2005; Klammer 2006) werden als Finanzierungsmodell Ziehungsrechte aus der Rentenanwartschaft vorgeschlagen. Die Rentenanwartschaft wäre nicht mehr in einem Stück 45 Jahre lang zu erbringen, aus ihr könnten zeitlich vorgezogen „Anteile“ entnommen werden. Die Weiterentwicklung solcher Modelle steht an, ohne dabei jedoch die Probleme gerade aus der GenderPerspektive zu vernachlässigen (Klammer 2006). Das bedeutet unter anderem: Das Anrecht auf Optionszeiten, die wahlweise für Aufgaben der Pflege von Kindern und Angehörigen, aber auch für soziale Tätigkeiten oder Weiterbildung genommen werden können, muss geschlechtsneutral formuliert und gestaltet sein. Solche Optionszeiten würden ihre logische Entsprechung etwa in modularisierten Ausbildungsgängen finden. Durch Optionszeiten werden Unterbrechungen im Lebenslauf normalisiert und neue Verknüpfungen von Tätigkeitsbereichen über die ganze Lebensspanne hinweg möglich. Dies trägt nicht zuletzt der längeren Lebenserwartung Rechnung, die "gewonnenen Jahre" können anders genutzt werden.

Ein zweites Lebenslaufmodell, das „Berufsanreicherungsmodell“, richtet sich auf die Restrukturierung von Berufsverläufen. Über neue Verknüpfungen von Erstausbildung, Optionszeiten und Erwerbsunterbrechungen sowie per Anrechnungsmöglichkeiten von Berufsausbildungen und Umstiegsausbildungen sollen neue Berufswege eröffnet werden. Insbesondere auf 
Frauen zielende Sackgassenkonstruktionen von Berufen sollen durch den Einstieg in die Modularisierung beruflicher Bildung verändert werden.

Die dritte Variante, das „Wunschzeitenmodell“, zielt darauf, die Lebensspanne, in der Kinderwünsche realisiert werden können, durch verbesserte Infrastrukturangebote zu erweitern. Hier geht es sowohl um die Möglichkeiten für junge Frauen beispielsweise ihre Ausbildung und das Großziehen von Kindern besser kombinieren zu können, was im Übrigen auch die Geburtenrate erhöhen könnte. So belegen europäische Studien, dass Mütter umso mehr Kinder gebären, je jünger sie bei der Erstgeburt sind (Ruckdeschel 2004). Aber auch eine biografisch späte Mutter-/Vaterschaft im Anschluss an eine kontinuierliche und intensive Vollzeitberufstätigkeit soll unterstützt werden.

\subsection{SZENARIO INFRASTRUKTUR}

Das zweite Szenario bezieht sich auf Infrastrukturen für Familien, also auf das Netz familiennaher Institutionen wie Schule, Geschäfte, Behörde etc., deren Organisation mit den Bedarfen von Familien - sei es als Eltern oder Arbeitnehmer - abzustimmen ist. Auch hier wird ein Perspektivenwechsel vollzogen: Eltern werden nicht als Nehmende, sondern als Investoren in den sozialen Nahraum betrachtet, die neue Entwicklungen anstoßen und realisieren wie z. B. Wohn- und Arbeitsformen, in denen Leben und Arbeiten für die verschiedenen Generationen näher zusammengebracht werden. Hierfür wäre die Stadtentwicklungs- und Gewerbeansiedlungspolitik umzuorientieren.

Im Hinblick auf Kinderbetreuung geht es um passfähigere und flexiblere Angebote sowie um neue Verbundsysteme der Kinderbetreuung, die Einrichtungen, Tagespflege, Initiativen und Ehrenamt zu einem gelingenden und qualitativ hochwertigen Ganzen zusammenbinden. Dies geschieht keinesfalls nur im Sinne einer besseren Vereinbarkeit für Eltern, sondern auch für eine bessere Förderung von Kindern in kleiner werdenden und zunehmend belasteten Familien. Neue Strukturen und Mischungen sind zu entwickeln, die über die klassische Institutionenlogik ebenso hinausgehen wie über die abgegrenzten Handlungslogiken von Professionellen, Semiprofessionellen und Laien. Beispiele gibt es bereits: Die sogenannten Familien- oder
Eltern-Kindzentren und neuerdings auch die Mehrgenerationenhäuser. Sie bieten idealtypisch - eine Vielfalt von Hilfen, Beratung, Bildung, Information und Kommunikation für alle Generationen an einem Ort. Sie sind mehr als die Summe von Angeboten, sie haben ein Konzept, das sich auf die besonderen Bedarfe der Region, der Kommune und des Viertels richtet und auch die Schwellen für schwer erreichbare, sozial benachteiligte Zielgruppen senkt. Ein weiteres Element ist der Ausbau familiennaher Dienstleistungen, der das Leben von Familien erheblich erleichtern kann wenn sie die Nutzung bezahlen können. "Mehr Familie durch weniger Familie“ durch den Ausbau von Infrastruktur - dies ist ein Konzept, das zwar das besonders deutsche Privatheitsdogma irritiert, aber in anderen Ländern zum Gedeihen von Familien beiträgt.

\subsection{SZENARIO GELD}

Damit ist das dritte Szenario "Geld“ im Blick. Geld ist eine unverzichtbare Ressource, um in einer Marktgesellschaft zu überleben, aber auch, um die besonderen Belastungen von Familien zumindest teilweise zu kompensieren. Wenig bekannt ist, dass Deutschland im Hinblick auf die finanzielle Förderung von Familien im europäischen Vergleich sogar etwas über dem Durchschnitt liegt. Deshalb geht es weniger um mehr Geld für Familien als um seine passgenaue Verteilung an spezifische Gruppen zu kritischen Zeitpunkten in Familienphasen. Hierfür wird angedacht, durch eine Familienkasse die derzeit herrschende Zersplitterung in den Zuständigkeiten für einzelne familienpolitische Maßnahmen und ihre Intransparenz zu beseitigen. Die Familienkasse soll zum einen den Zugang zu Leistungen vereinfachen, indem Familien einen zentralen Ansprechpartner haben. Zum anderen soll sie die finanzielle Förderung von Familien institutionell stärken. Weitere Diskussionspunkte sind: Die Vereinfachung des Systems steuerlicher Leistungen für Familien sowie das einkommensabhängige Elterngeld, das bereits zum 1.1.2007 eingeführt wurde und das die zunächst vielfältig diffamierten Vätermonate regelt. ${ }^{1}$

\section{Der Siebte Familienbericht: Feministische Familien- politik oder konservativer Feminismus?}

Alle drei Szenarien sind, wenn auch in unterschiedlicher Weise, relevant für eine bessere Gleichstellung der Geschlechter: Direkt durch die Zielsetzung des ZweiverdienerFürsorge-Modells für beide Geschlechter bei der Neugestaltung von Arbeits- und Alltagszeit sowie der Lebensverläufe. Auch die Betonung der Rolle des fürsorglichen Vaters ist hier relevant. In eher indirekter, geschlechtsneutraler Weise wirken Infrastrukturen, die den Alltag für Eltern erleichtern, und die passgenauere Lenkung von finanziellen Transferleistungen für Familien.

Eine derartige Fokussierung der Politik auf Familie lässt sich schwerlich als antifeministisch bezeichnen, zumal die Wünsche und Einstellungen junger Frauen und Männer sich nach wie vor und sogar zunehmend positiv auf Familie, Kinder und Liebe richten (Cornelißen 2006). Ein Fünftel der jungen Frauen setzt vorwiegend auf Familie, ein zweites Fünftel vorwiegend auf Beruf (Hakim 2003; Keddi et al. 1999), drei Fünftel wünschen sich ihre Verbindung; allerdings können sich diese Verteilungen im Lebenslauf verschieben, sie sollten also nicht statisch gesetzt werden. Die vorgeschlagenen Maßnahmen beziehen sich schwerpunktmäßig auf diese drei Fünftel sowie auf die Öffnung fürsorgeorientierter Lebensmodelle auch für Männer. Für Deutschlands Familienpolitik stellt dieser Bericht aufgrund der Integration gleichstellungsrelevanter Aspekte eine kleine Re-

\footnotetext{
Diese Regelung hat den Anteil aktiv betreuender Väter bis zum 15.8.2007 im Bundesdurchschnitt bereits von 3,5 auf 8,5\% erhöht (BMFSFJ 2007). Allerdings ist die Elternzeit ohne eine an das erste Lebensjahr des Kindes anschließende ausreichende öffentliche Kinderbetreuung und ohne realisierbare Arbeitsplatzgarantien für Eltern ein zahnloser Tiger, sie kann sogar im Hinblick auf Geschlechtergerechtigkeit einen "backlash" nach sich ziehen. Bislang sind die Fortschritte beim Ausbau der Betreuung für unter Dreijährige noch sehr mäßig, für nur 8 \% der unter Dreijährigen in Westdeutschland gab es 2006 Betreuungsplätze in Einrichtungen oder Tagespflege (DJ 2007). Dies verteilt sich zudem auf die westlichen Bundesländer sehr unterschiedlich, manche ländlichen Regionen sind völlig unterausgestattet.
} 
volution dar, wird doch endlich mit dem kulturell lange gültigen Muster der guten verhäuslichten Mutter gebrochen. Vielleicht liegt der größte Gewinn des Familienberichts darin, dass er zur Akzeptanz der kulturellen Sozialfigur der erwerbstätigen Mutter beiträgt und hierfür fundierte strukturelle Vorschläge unterbreitet. Würde der im Familienbericht vorgeschlagene Weg konsequent und mit Hilfe konkretisierter Maßnahmebündel verfolgt, so könnte die im Vergleich zu vielen europäischen Ländern offensichtliche Modernisierungslücke der deutschen Familienpolitik ein Stück weit geschlossen werden.

Zugleich werden hier die Grenzen des emanzipatorischen Ansatzes des Familienberichts deutlich. Sie sind erstens darauf zurückzuführen, dass neben den fürsorgebezogenen Schnittmengen von Gleichstellungs- und Familienpolitik zu Recht deutliche Unterschiede zwischen ihnen bestehen bleiben (Jurczyk 1993). Die fortbestehende soziale Ungleichheit von Frauen z. B. in der Lohnlücke von ca. $25 \%$, ihre niedrigere Position im beruflichen und gesellschaftlichen Statusgefüge (Cornelißen 2005), die Gewalt gegen Frauen und anderes mehr - verweisen auf Diskriminierung aufgrund des Geschlechts und nicht aufgrund der Einbindung von Frauen in Fürsorgearbeit.

Zum zweiten bleibt in dem Bericht die Vielfalt weiblicher Lebensentwürfe verengt auf ein Leben in Fürsorgebeziehungen, wenn auch nicht mehr ausschließlich auf die sogenannte Normalfamilie fokussiert. Dieser Blickwinkel verdankt sich zwar der Themenstellung des Berichts als Familienbericht, in den anschließenden öffentlichen Debatten wird jedoch Familie zum Wert an sich; der Sinn eines Lebens ohne Kinder (und Mann) und die positive Besetzung anderer Lebens- und Beziehungsformen geraten allzu leicht aus dem Blick (Eckart
2007). In diesen beiden Aspekten liegt die eigentliche Gefahr der derzeitigen Ungleichgewichtigkeit von Gleichstellungsund Familienpolitik. Der „konservative Feminismus", der der derzeitigen Familienund Frauenministerin unterstellt wird (FAZ.Net 2007), hat in dieser latent normativen Zuspitzung seine berechtigte Wurzel, ist allerdings nicht im Bericht angelegt.

Drittens zeigen sich Mängel, die nur durch eine feministische Familienpolitik zu beheben wären, die über Gleichstellung und Emanzipation von Frauen hinausgeht (Stolz-Willig 2004). Feministische Familienpolitik umfasst stets Herrschaftskritik, d.h. Kritik an Hierarchien und Ungleichheiten unterschiedlicher Art. Schwachstellen hat der Bericht deshalb dort, wo es um die ökonomische Abhängigkeit von Frauen geht, die auch durch deren erweiterte Teilhabe im Rahmen von Teilzeitarbeit nicht wirklich behoben wäre. Über den Bericht hinausgehende Forderungen wären im Szenario „Geld“ deshalb bspw. die konsequente Einführung der Individualbesteuerung nach skandinavischem Modell und die steuerrechtliche De-Privilegierung der Ehe (Mückenberger et al. 2007; Spangenberg 2005), aber auch Vorschläge zu einer konsequenteren Verbesserung der Situation Alleinerziehender. In der Fokussierung auf das Zweiverdiener-Fürsorgemodell geht dies - wenig überraschend - verloren. Zudem - auch hier wären die beiden Szenarien Infrastruktur und Geld weiter zu entwickeln - ist der Blick auf Familien von einer latenten Mittelschichtorientierung geprägt. Zwar werden die häufig mit Migrationshintergrund verbundenen Armutsrisiken von Familien im Bericht kritisch analysiert, sie sind aber kaum Gegenstand von Vorschlägen. Jedoch ist die wachsende, auch regional fassbare, soziale Ungleichheit zwischen Arm und Reich und die damit verbundene Polarisierung zwi- schen privilegierten und de-privilegierten Familien (Zeitschrift für Familienforschung 2005) - auch in ihren Folgen für die kognitive, gesundheitliche, emotionale und soziale Entwicklung von Kindern (Sann/ Thrum 2005) - eine mindestens ebenso große gesellschaftliche Herausforderung wie der demografische Wandel.

Feministische Familienpolitik müsste entlang des Ansatzes der Intersektionalität (Crenshaw 1994) solche Ungleichheitsdimensionen auch in ihrer wechselseitigen Überlagerung zum Ausgangspunkt nehmen.

Der Siebte Familienbericht ist ein Beitrag zum Abbau sowohl des Antifeminismus der Familienpolitik als auch des Antifamilialismus der Frauengleichstellungspolitik. Damit kann das in Deutschland stets umstrittene Thema Familie einen Normalisierungsschub jenseits von konservativen oder links-feministischen Ideologien erfahren. Überdies würde die Umsetzung des Leitbildes einer geschlechter- und generationengerechten Familie zu einer Annäherung an europäische Nachbarländer führen.

Ein weiteres Problem allerdings bleibt: Denn selbst die noch in weiter Ferne befindliche Realisierung des egalitären Zweiverdiener-Fürsorgemodells kann nur der notwendige Ausgangspunkt für neue Utopien gerechter Lebenschancen und für ein "gutes Leben" sein. Wir müssen darauf achten, dass das vorgeschlagene Modell angesichts einer immer dominanter werdenden marktförmigen Arbeitsgesellschaft wenn auch nicht zur Ulrich Beck'schen Vision von vollmobilen Single-Arbeitsmonaden (Beck 1986), so dann doch zur schlechten Realität der von Arlie Hochschild (2002) geschilderten vollflexiblen überarbeiteten Elternpaare führt. Ein solcher Preis für die Überwindung traditioneller und ungerechter Lebensformen wäre (zu) hoch.

\section{LITERATUR}

Auth, D./Holland-Cunz, B. (Hrsg.) (2007): Grenzen der Bevölkerungspolitik. Strategien und Diskurse demographischer Steuerung, Opladen/ Farmington Hills

Beck, U. (1986): Risikogesellschaft. Auf dem Weg in eine andere Moderne, Frankfurt/Main
Becker-Schmidt, R. (2004): Doppelte Vergesellschaftung von Frauen: Divergenzen und Brückenschläge zwischen Privat- und Erwerbsleben, in: Becker, R./Kortendiek, B. (Hrsg.): Handbuch Frauen- und Geschlechterforschung. Theorie, Methoden, Empirie, Wiesbaden, S. 62-71 Bertram, H./Rösler, W./Ehlert, N. (2005): Nachhaltige Familienpolitik. Zukunftssicherung durch einen Dreiklang von Zeitpolitik, finanzieller Transferpolitik und Infrastrukturpolitik, Berlin 
Bien, W./Marbach, J. (2003): Partnerschaft und Familiengründung, Opladen

Björklund, A. (2007): Does a family-friendly policy affect fertility? Swedish Institute for European Policy Studies 3, Stockholm Bundesministerium für Familie, Senioren, Frauen und Jugend (BMFSFJ) (2006): Familie zwischen Flexibilität und Verlässlichkeit. Perspektiven für eine lebenslaufbezogene Familienpolitik. Siebter Familienbericht, Berlin Bundesministerium für Familie, Senioren, Frauen und Jugend (BMFSFJ) (2007): „Elterngeld erfüllt alle Erwartungen“ , Pressemitteilung, 15.8 Cornelißen, W. (Hrsg.) (2005): Datenreport zur Gleichstellung von Frauen und Männern in der Bundesrepublik Deutschland; im Auftrag des BMFSFJ, München

Cornelißen, W. (2006): Kinderwunsch und Kinderlosigkeit im Modernisierungsprozess, in: Berger, P. A./Kahlert, H. (Hrsg.): Der demographische Wandel. Chancen für die Neuordnung der Geschlechterverhältnisse, Frankfurt/New York, S. 137-163

Crenshaw, K. W. (1994): Mapping the Margins: Intersectionality, Identity Politics, and Violence Against Women of Color, in: Fineman, Martha Albertson/Mykitiuk, Rixanne (Hrsg.): The Public Nature of Private Violence, New York, S. 92-114

Deutsche Gesellschaft für Zeitpolitik (DGFZP) (2005): Zeit ist Leben, Zeitpolitisches Manisfest, Bremen

Deutsches Jugendinstitut (DJI) (Hrsg.) (2007): Untersuchung zum Ausbau der Kindertagesbetreuung für unter 3-jährige Kinder; internes Arbeitspapier, München

Eckart, Ch. (2007): Privatheit - Zur Gestaltung von Beziehungen des Sorgens, in: Jurczyk, K./Oechsle, M. (Hrsg.): Das Private neu denken. Erosionen, Ambivalenzen, Leistungen, Münster, im Erscheinen

Eichhorst, W./Kaiser, L./Thode, E./Tobsch, V. (2007): Vereinbarkeit von Familie und Beruf im internationalen Vergleich. Zwischen Paradigma und Praxis, Gütersloh

FAZ.Net (Frankfurter Allgemeine Zeitung) (2007): Konservativer Feminismus ist ein spannender Begriff. Interview mit Frau von der Leyen, 19.3. Hakim, C. (2003): Models of the Family in Modern Societies. Ideals and Realities, Aldershot

Hochschild, A. R. (2002): Keine Zeit. Wenn die Firma zum Zuhause wird und zu Hause nur Arbeit wartet, Opladen

Holst, E. (2007): Arbeitszeitwünsche von Frauen und Männern liegen näher beieinander als tatsächliche Arbeitszeiten, in: Deutsches Institut für Wirtschaftsforschung 14/15, S. 209-215

Hondrich, K. O. (2006): Der Neue Mensch, Frankfurt/Main

Jurczyk, K. (1976): Frauenarbeit und Frauenrolle. Zum Zusammenhang von Familienpolitik und Frauenerwerbstätigkeit in Deutschland von 1918-1975, München

Jurczyk, K. (1993): Flexibilisierung für wen? Zum Zusammenhang von Arbeitszeiten und Geschlechterverhältnissen, in: Jurczyk, K./Rerrich, M. S. (Hrsg.): Die Arbeit des Alltags. Beiträge zu einer Soziologie der alltäglichen Lebensführung, Freiburg, S. 346-374

Jurczyk, K./Lange, A./Szymenderski, P. (2005): Zwiespältige Entgrenzungen: Chancen und Risiken neuer Konstellationen zwischen Familien- und Erwerbstätigkeit, in: Mischau, A./Oechsle, M. (Hrsg.): Arbeitszeit - Familienzeit - Lebenszeit: Verlieren wir die Balance? Wiesbaden, S. 13-33 Jurczyk, K./Schier, M. (2007): Familie als Herstellungsleistung in Zeiten der Entgrenzung, in: Aus Politik und Zeitgeschichte 34, S. 10-17

Kaufmann, F.-X. (2005): Die schrumpfende Gesellschaft, Frankfurt/Main Keddi, B./Pfeil, P./Strehmel, P./Wittmann, S. (1999): Lebensthemen junger Frauen. Die andere Vielfalt weiblicher Lebensentwürfe, Opladen
Klammer, Ute (2006): Zeit, Geld und soziale Sicherung im Lebensverlauf - Empirische Befunde als Herausforderung für die Gestaltung einer lebensbegleitenden Familien- und Sozialpolitik, in Bertram, H./Krüger, H./Spieß, C. K. (Hrsg.): Wem gehört die Familie der Zukunft? Expertisen zum Siebten Familienbericht der Bundesregierung, Opladen/Farmington S. $423-455$

Klein, Th. (2005): Sozialstrukturanalyse. Eine Einführung, Reinbek

Klenner, Ch. 2007: Familiengerechte Arbeitszeiten. Präsentation bei der Hans-Böckler-Stiftung, 31.5., Düsseldorf

Krüger, H. (2005): Kulturelle Ungleichheit, Institutionen des Lebenslaufs und die Zukunft der Geschlechterdifferenz, in: Rehberg, K.-S.(Hrsg.):

Soziale Ungleichheit, kulturelle Unterschiede. Verhandlungen des 32.

Kongresses der Deutschen Gesellschaft für Soziologie in München 2004, Teil 1, Frankfurt/Main, S. 144-158

Krüger, H. (2006): Geschlechterrollen im Wandel - Modernisierung der Familienpolitik, in: Bertram, H./Krüger, H./Spieß, C. K. (Hrsg.): Wem gehört die Familie der Zukunft? Expertisen zum Siebten Familienbericht der Bundesregierung, Opladen/Farmington, S. 191-206

Lange, A./Bräuninger, B./Lüscher, K. (2000): Der Wandel von Familie. Zur Rhetorik sozialwissenschaftlicher Texte, in: Österreichische Zeitschrift für Soziologie 1, S. 3-28

McDonald, P. (2000): The Toolbox of Public Policies to Impact on Fertility - A Global View. Paper presented at the seminar "Low fertility, families and public policies", Sevilla, 15.-16. 09.

Meier, U./Preusse, H./Sunnus, E. M. (2003): Steckbriefe von Armut. Haushalte in prekären Lebenslagen, Wiesbaden Mills, M./Blossfeld, H.-P. (2003): Globalization, Uncertainty and Changes in Early Life Courses, in: Zeitschrift für Erziehungswissenschaft 2, S. $188-218$

Mückenberger, U./Spangenberg, U./Warncke, K. (2007): Familienförderung und Gender Mainstreaming im Steuerrecht, Baden-Baden Ruckdeschel, K. (2004): Determinanten des Kinderwunsches in Deutschland, in: Zeitschrift für Bevölkerungsforschung 3/4, S. 363-386 Sann, A./Thrum, K. (2005): Opstapje - Schritt für Schritt. Abschlussbericht des Modellprojekts, München

Schier, M./Lange, A. (2007): Familien heute: Strukturelle Trends und alltägliche Realitäten, in: Prävention - Zeitschrift für Gesundheitsförderung 2, S. 35-39

Spangenberg, U. (2005): Neuorientierung der Ehebesteuerung: Ehegattensplitting und Lohnsteuerverfahren. Hans-Böckler-Stiftung, Arbeitspapier 106, Düsseldorf

Stauber, B./du Bois-Reymond, M. (2006): Familienbeziehungen im Kontext verlängerter Übergänge. Eine intergenerative Studie aus neun europäischen Ländern, in: Zeitschrift für Soziologie der Erziehung und Sozialisation 2, S. 206-221

Stolz-Willig, B. (2004): Familienpolitik - Leitbilder, Realitäten und Modernisierungsbedarfe, in: Forum Erwachsenenbildung 1, S. 15-20

Tölke, A. (2004): Die Bedeutung von Herkunftsfamilie, Berufsbiografie und Partnerschaften für den Übergang zur Ehe und Vaterschaft, in: Tölke, A./Hank, K. (Hrsg.): Männer - das "vernachlässigte" Geschlecht in der Familienforschung, Wiesbaden, S. 98-126

Tronto, J. (2000): Demokratie als fürsorgliche Praxis, in: Feministische Studien extra 18, S. 25-42

Zeitschrift für Familienforschung (2005): Die ökonomische Situation von Familie 2 